\title{
Galactose-modified duocarmycin prodrugs as senolytics
}

\author{
Ana Guerrero $^{1,2}$ | Romain Guiho ${ }^{3}$ | Nicolás Herranz ${ }^{1,2}$ | Anthony Uren ${ }^{1,2}$ | \\ Dominic J. Withers $^{1,2}$ | Juan Pedro Martínez-Barbera ${ }^{3}$ | Lutz F. Tietze $^{4}$ | Jesús Gil ${ }^{1,2}$
}

\author{
${ }^{1} \mathrm{MRC}$ London Institute of Medical Sciences \\ (LMS), London, UK \\ ${ }^{2}$ Faculty of Medicine, Institute of Clinical \\ Sciences (ICS), Imperial College London, \\ London, UK \\ ${ }^{3}$ Developmental Biology and Cancer \\ Programme, Birth Defects Research Centre, \\ Great Ormond Street Institute of Child \\ Health, University College London, London, \\ UK \\ ${ }^{4}$ Institute of Organic and Biomolecular \\ Chemistry, Georg-August University, \\ Göttingen, Germany

\section{Correspondence} \\ Jesús Gil, Faculty of Medicine, Institute \\ of Clinical Sciences (ICS), Imperial College \\ London, Du Cane Road, London W12 ONN, \\ UK. \\ Email: jesus.gil@imperial.ac.uk \\ Funding information \\ Medical Research Council, Grant/ \\ Award Number: MC-A652-5PZ00, \\ MC_U120085810 and MC-A654-5QB40; \\ Wellcome Trust, Grant/Award Number: \\ 098565; Brain Tumour Charity; Children \\ with Cancer UK; CRUK; Great Ormond \\ Street Hospital Charity; NIHR Biomedical \\ Research Centre at University College \\ London Hospitals NHS Foundation Trust and \\ University College London
}

\begin{abstract}
Senescence is a stable growth arrest that impairs the replication of damaged, old or preneoplastic cells, therefore contributing to tissue homeostasis. Senescent cells accumulate during ageing and are associated with cancer, fibrosis and many age-related pathologies. Recent evidence suggests that the selective elimination of senescent cells can be effective on the treatment of many of these senescence-associated diseases. A universal characteristic of senescent cells is that they display elevated activity of the lysosomal $\beta$-galactosidase, and this has been exploited as a marker for senescence (senescence-associated $\beta$-galactosidase activity). Consequently, we hypothesized that galactose-modified cytotoxic prodrugs will be preferentially processed by senescent cells, resulting in their selective killing. Here, we show that different galactose-modified duocarmycin (GMD) derivatives preferentially kill senescent cells. GMD prodrugs induce selective apoptosis of senescent cells in a lysosomal $\beta$-galactosidase (GLB1)-dependent manner. GMD prodrugs can eliminate a broad range of senescent cells in culture, and treatment with a GMD prodrug enhances the elimination of bystander senescent cells that accumulate upon wholebody irradiation treatment of mice. Moreover, taking advantage of a mouse model of adamantinomatous craniopharyngioma (ACP), we show that treatment with a GMD prodrug selectively reduced the number of $\beta$-catenin-positive preneoplastic senescent cells. In summary, the above results make a case for testing the potential of galactose-modified duocarmycin prodrugs to treat senescence-related pathologies.
\end{abstract}

\section{KEYWORDS}

duocarmycin, prodrug, senescence, senescence-associated $\beta$-galactosidase, senolytics

\section{1 | INTRODUCTION}

Cellular senescence is a stress response that prevents the replication of old, damaged or transformed cells (Herranz \& Gil, 2018). Senescence can be induced by replicative exhaustion and also by a range of insults that includes oncogenic activation, genotoxic stress 
or SASP, which is a prominent mediator of the patho-physiological effects of senescence (Acosta et al., 2008, 2013; Coppe, Desprez, Krtolica, \& Campisi, 2010; Kuilman \& Peeper, 2009).

Despite that the acute induction of senescence limits fibrosis and protects against cancer progression, the abnormal accumulation of senescent cells with age or in diseased tissues is detrimental (Munoz-Espin \& Serrano, 2014). Interestingly, evidence drawn from genetic models has shown that eliminating senescent cells increases lifespan, improves healthspan and benefits the outcomes of a wide range of diseases (Baker et al., 2011, 2016; Childs et al., 2016, 2017). These studies have led to a collective effort to identify "senolytics," drugs that selectively kill senescent cells. Several senolytics have been identified including dasatinib and quercetin (Zhu et al., 2015), piperlongumine (Wang et al., 2016), FOXO4-interfering peptides (Baar et al., 2017), HSP90 inhibitors (Fuhrmann-Stroissnigg et al., 2017), cardiac glycosides (Guerrero et al., 2019; Triana-Martinez et al., 2019) or the Bcl2 family inhibitors ABT-263 (navitoclax) and ABT-737 (Chen et al., 2015; Yosef et al., 2016; Zhu et al., 2016). Currently, Bcl2 family inhibitors have become the gold standard on senolysis. Bcl2 family inhibitors eliminate a range of senescent cells in vivo and reproduce the effects observed in transgenic mice modelling senescence ablation (Ovadya \& Krizhanovsky, 2018). However, ABT263 causes severe thrombocytopenia and neutropenia, what might complicate its use on the clinic. Moreover, it is becoming evident that specific senolytics might be necessary to eliminate different types of senescent cells. Therefore, there is a need to identify additional drugs with senolytic properties.

An alternative strategy for targeting senescence is to exploit properties that differentiate senescent from normal cells. In this regard, the senescence-associated $\beta$-galactosidase activity (SA- $\beta$-gal) is one of the more conserved and defining characteristics of senescent cells. Senescent cells present an increased lysosomal mass (Kurz, Decary, Hong, \& Erusalimsky, 2000). As a result, senescent cells display elevated levels of lysosomal enzymes such as $\beta$-galactosidase (encoded by GLB1 (Dimri et al., 1995)) or $\alpha$-fucosidases (Hildebrand et al., 2013). Indeed, it has been shown that galacto-oligosaccharide encapsulated nanoparticles (GalNP) preferentially release their content on senescent cells (Agostini et al., 2012). Consequently, this GalNP can be used in combination with different cargos to either image or kill senescent cells (Munoz-Espin et al., 2018).

Galactose modification has been frequently used to improve the pharmacokinetic properties or the delivery of existing drugs. In addition, galactose modification can be used to generate prodrugs that rely on E. coli $\beta$-galactosidase for controlled activation (Melisi, Curcio, Luongo, Morelli, \& Rimoli, 2011). When combined with antibody-linked $\beta$-galactosidase, this approach is known as antibody-directed enzyme prodrug therapy (ADEPT) (Bagshawe, 2006; Tietze \& Schmuck, 2011). In ADEPT, a conjugate of a tumour-specific antibody and an enzyme, such as $\beta$-galactosidase, is combined with the application of a hardly cytotoxic prodrug. By means of the enzyme in the conjugate, the prodrug is selectively cleaved in cancer cells leading to the formation of a highly cytotoxic compound. Several of these galactose-modified cytotoxic prodrugs have been described (Leenders et al., 1999). A class of such prodrugs are galactose-modified duocarmycin (GMD) derivatives (Tietze, Major, \& Schuberth, 2006). Duocarmycins are a group of antineoplastic agents with low picomolar potency. They are thought to act by binding and alkylating double-stranded DNA in AT-rich regions of the minor groove (Boger, Johnson, \& Yun, 1994; Tietze et al., 2006; Tietze, Schuster, Krewer, \& Schuberth, 2009), but alternative mechanisms of action have been proposed to account for the cytotoxic effects of duocarmycin dimers (Wirth, Schmuck, Tietze, \& Sieber, 2012).

Here, we investigated whether galactose-modified prodrugs can preferentially kill senescent cells. We have assessed several GMD derivatives and confirmed their senolytic potential in cell culture, ex vivo and in vivo. Given the increasing list of senescence-associated diseases and the benefits of senolytic treatment, we propose that GMD derivatives and, more generally, galactose-modified prodrugs are a new class of senolytic compounds and they should be tested to assess their therapeutic potential.

\section{2 | RESULTS}

\section{1 | A galactose-modified duocarmycin prodrug with senolytic properties}

The natural antibiotic duocarmycin is a highly cytostatic compound (Boger \& Johnson, 1995). A series of glycosidic derivatives of duocarmycin have been previously developed to be used as prodrugs in the context of antibody-directed enzyme prodrug therapy (ADEPT) (Tietze, Hof, Muller, Krewer, \& Schuberth, 2010; Tietze et al., 2009). Given that senescent cells display elevated levels of SA- $\beta$ galactosidase activity, we hypothesized that galactose-modified cytotoxic prodrugs will be preferentially processed by senescent cells, resulting in their selective killing. To test this hypothesis, we took advantage of a galactose-modified duocarmycin (GMD) prodrug (referred as prodrug A, JHB75B) previously described (Tietze et al., 2009). We analysed the effects that a seco-duocarmycin analogue dimer (duocarmycin SA) and its galactose derivative (prodrug A) had on the survival of IMR90 ER:RAS cells, a model of oncogene-induced senescence (OIS). Activation of the ER:RAS fusion with 4-hydroxytamoxifen (4OHT) induces senescence in IMR90 ER:RAS cells (Georgilis et al., 2018). Treatment with duocarmycin SA was equally effective in killing normal and senescent cells, with the exception of a small selectivity towards senescent cells at the lower concentrations (Figure 1a). In contrast, when we treated IMR90 ER:RAS cells with prodrug $A$ (differing only in the addition of two galactose groups that inactivate it), we observed the preferential elimination of senescent cells (Figure $1 \mathrm{~b}$ and Figure S1a). Duocarmycins are known to bind and alkylate DNA in AT-rich regions of the minor groove and induce cell death in a way dependent of DNA replication (Boger et al., 1994; Tietze et al., 2006, 2009) We checked that senescent cells were growth arrested at the time of the drug treatment (Figure S1b). This shows that the effect observed is not due to hyperreplication of cells during early stages of OIS and suggests that the prodrug might 
(a)<smiles>O=C(CCCC(=O)N1CC(CCl)c2cc(O)c3ccccc3c21)N1Cc2cc(O)c3ccccc3c2C(CCl)C1</smiles>

(b)

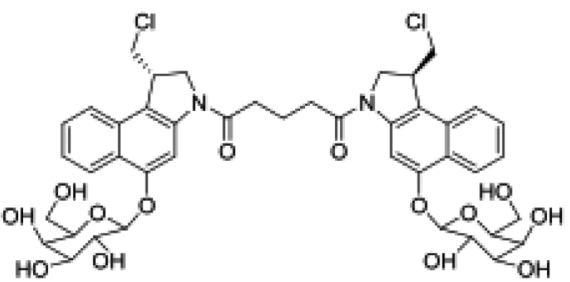

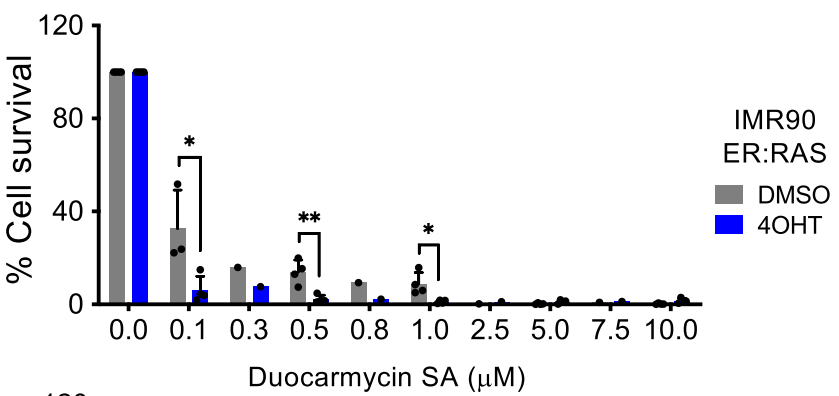

(c)

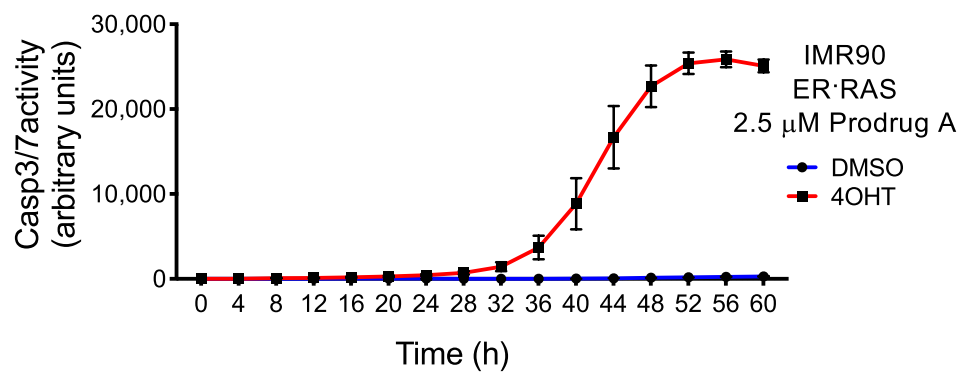

(d)

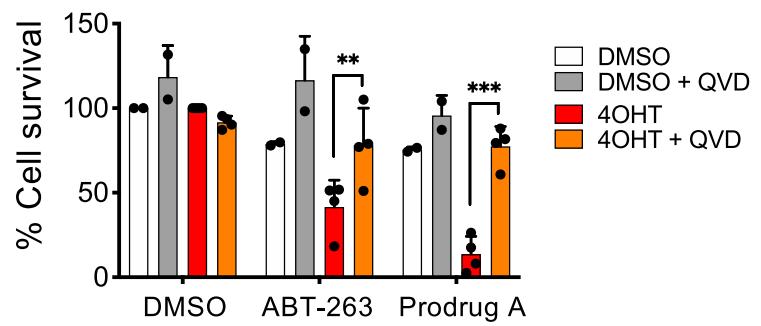

FIGURE 1 A galactose-modified duocarmycin prodrug with senolytic properties. (a) Molecular structure of seco-duocarmycin analog dimer (JHB71A, left). IMR90 ER:RAS cells were treated with DMSO or with 4OHT (4-hydroxy-tamoxifen) for 6 days to induce OIS. Cells were treated with the indicated concentrations of seco-duocarmycin analog dimer for $72 \mathrm{hr}$. Cell numbers were quantified using DAPI staining, and percentage of survival cells are plotted (right) $(n=4)$. (b) Molecular structure of a galactose-modified prodrug derivative of seco-duocarmycin analog dimer (JHB75B, referred as prodrug A, left). Cells were treated with prodrug A for $72 \mathrm{hr}$ as described before $(n=4)$. (c) Treatment of senescent cells with a GMD prodrug triggers caspase-3/7 activity. IMR90 ER:RAS were treated with $40 H T$ or vehicle (DMSO) for 6 days to induce senescence. $2.5 \mu \mathrm{M}$ prodrug was then added together with NucLight Rapid Red Reagent for cell labelling and Caspase-3/7 reagent for apoptosis (IncuCyte). Caspase 3/7 activity was measured at 4-hr intervals. (d) After 6-day treatment with 4OHT or vehicle (DMSO), IMR90 ER:RAS were treated with $1 \mu \mathrm{M} \mathrm{ABT-263} \mathrm{or} 2.5 \mu \mathrm{M}$ prodrug A for $72 \mathrm{hr}$ in the presence or absence of the pancaspase inhibitor Q-VD-OPh $(n=4)$. All statistical significances were calculated using unpaired Student's $t$ tests. All error bars represent mean \pm s.d; $n$ represents independent experiments.; ns, not significant; ${ }^{*} p<.05 ;{ }^{* *} p<.01 ;{ }^{* * *} p<.001,{ }^{* * * *} p<.0001$

act by some of the alternative cytotoxic mechanisms described for duocarmycin dimers (Wirth et al., 2012). Treatment with prodrug $A$ induced caspase $3 / 7$ activity on senescent cells (Figure $1 c$ ), and the selective death of these cells was prevented with a pan-caspase inhibitor (Figure 1d). The above results suggest that GMD prodrugs can behave as senolytics by selectively inducing apoptosis on senescent cells.

\subsection{Senolytic properties of prodrug A depend on the lysosomal $\beta$-galactosidase}

We had hypothesized that GMD prodrugs could behave as senolytics due to the higher SA- $\beta$-galactosidase activity of senescent cells. To investigate whether the levels of $\beta$-galactosidase activity correlate with the sensitivity of senescent cells to GMD prodrugs, we induced senescence in IMR90 ER:RAS cells. Afterwards, we treated control or senescent cells with $2.5 \mu \mathrm{M}$ prodrug $\mathrm{A}$ and used a fluorescent substrate (DDAO) to quantify SA- $\beta$-galactosidase activity at singlecell resolution (Figure 2a). IMR90 ER:RAS cells undergoing OIS had more cells with higher SA- $\beta$-galactosidase levels, as it was evident when analysing cell intensities (Figure $2 b$ ), or when using threshold system to quantify the percentage of SA- $\beta$-galactosidase-positive cells (Figure $2 \mathrm{c}$ ) or divide the cells in negative, high and highest for SA- $\beta$-galactosidase (Figure S2). While treatment with $2.5 \mu \mathrm{M}$ prodrug $A$ selective killed the majority of the senescent cells (Figure S1a), it was interesting to observe that the senescent cells that survived display lower levels of SA- $\beta$-galactosidase activity (Figure $2 a-c$ and 
(a)

DMSO

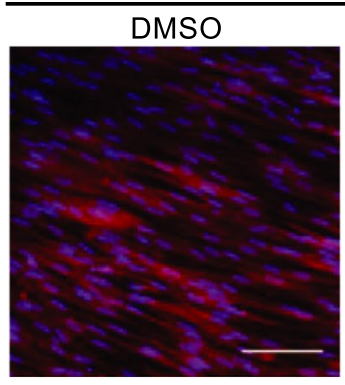

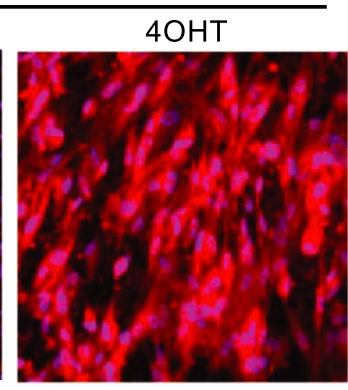

$2.5 \mu \mathrm{M}$ Prodrug A

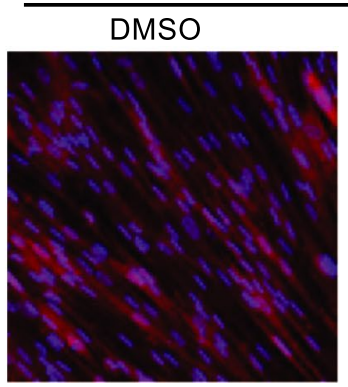

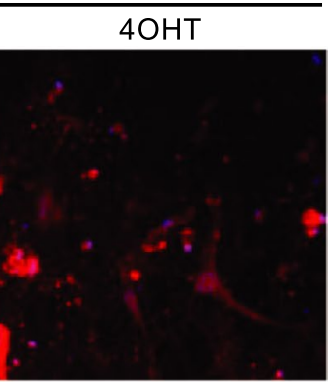

IMR90

ER:RAS

SA- $\beta-G a l$

(DDAO) (b)

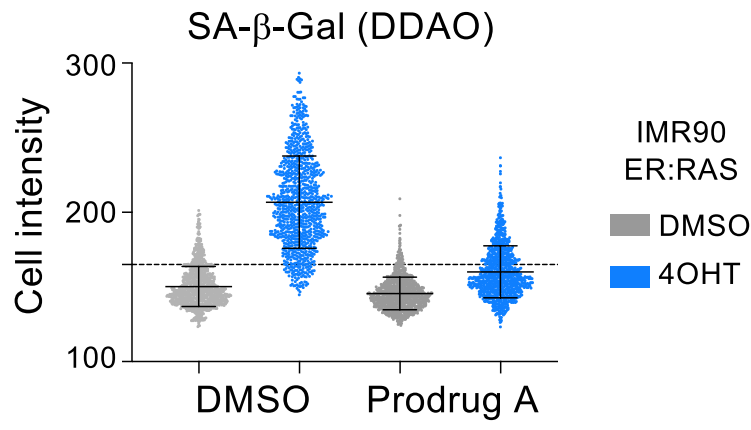

(c)

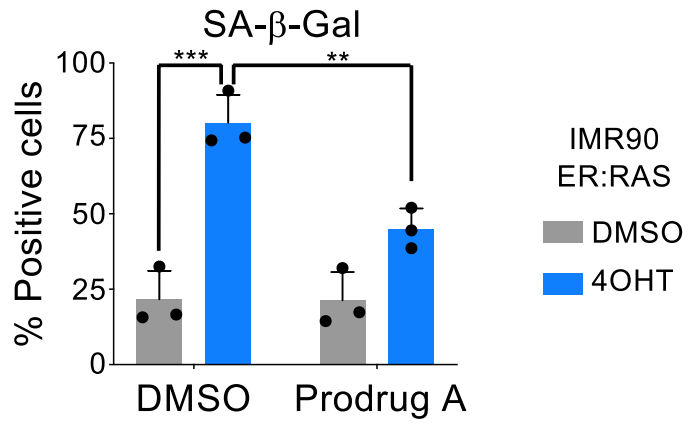

(d)

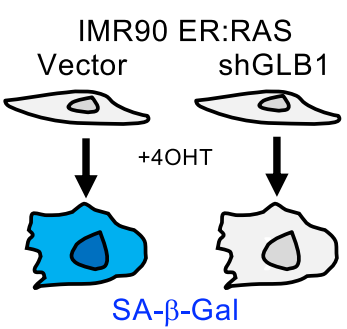

(f)

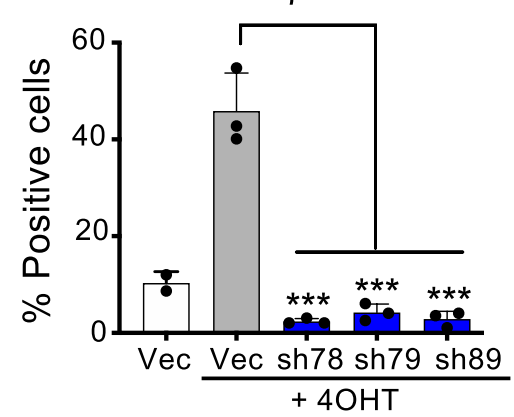

(e)

$+4 \mathrm{OHT}$

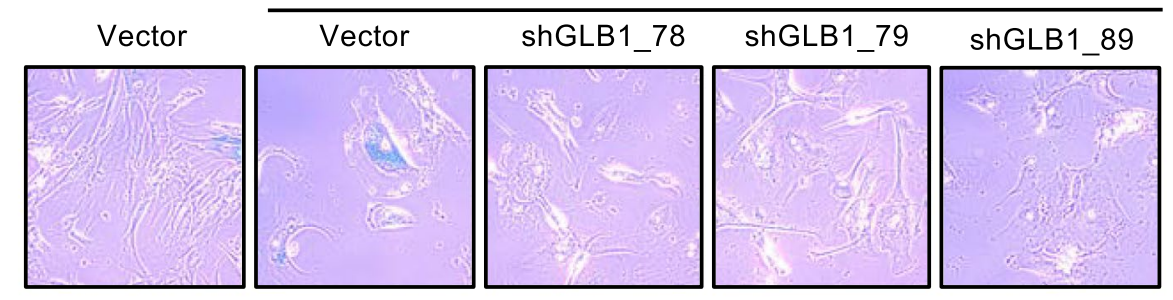

(g)
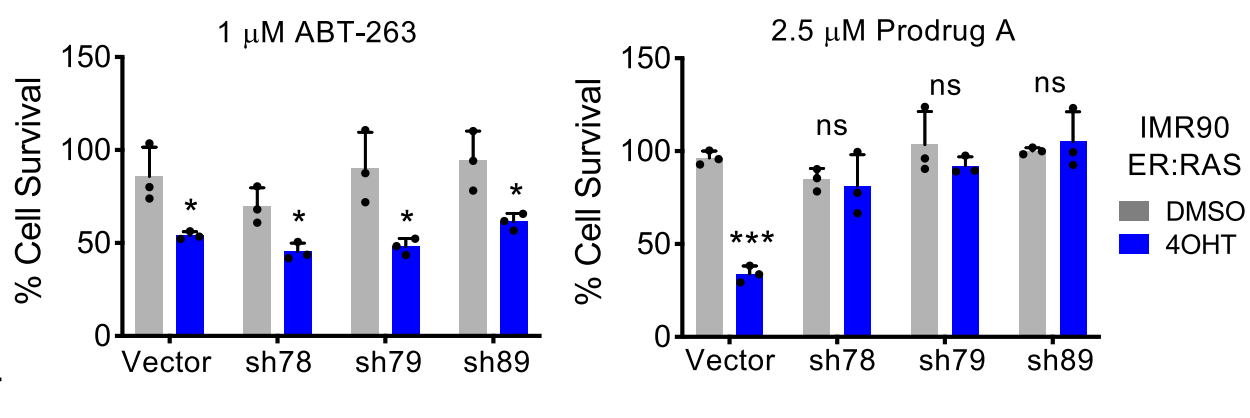

IMR90

ER:RAS

SA- $\beta-$ Gal

FIGURE 2 Galactose-modified duocarmycin prodrugs preferentially target senescent cells with high SA- $\beta$-galactosidase activity. (a) Representative pictures of fluorescent SA- $\beta$-galactosidase staining in IMR90 ER:RAS cells treated with prodrug A or vehicle. Scale bar, $100 \mu \mathrm{m}$. (b) Single-cell intensities value for DDAO galactoside in a representative well of a 96-well plate seeded with IMR90 ER:RAS cells treated with DMSO or $4 \mathrm{OHT}$. Grey dotted line indicates quantification cut-off. Cells were considered positives for SA- $\beta$-gal when their cell intensity was > 165. (c) Quantification of SA- $\beta$-galactosidase activity in IMR90 ER:RAS cells treated with prodrug A or vehicle. Statistical significance was calculated using two-way ANOVA. (d-g) The senolytic properties of prodrug A depend on the lysosomal $\beta$-galactosidase (GLB1). (d) Experimental set-up. (e) Representative pictures of cytochemical SA- $\beta$-Gal staining in IMR90 ER:RAS infected with different shRNAs against GLB1 or an empty vector and ( $f$ ) quantification $(n=3)$. Statistical significance was calculated using one-way ANOVA. (g) Quantification of cell survival of senescent and control IMR90 ER:RAS infected with different shRNAs targeting GLB1 or an empty vector and treated with ABT-263 or prodrug A for 3 days $(n=3)$. Statistical significance was calculated using two-tailed, Student's $t$ test. All error bars represent mean $\pm S D ; n$ represents independent experiments; ns, not significant; ${ }^{*} p<.05 ;{ }^{* *} p<.01 ;{ }^{* * *} p<.001,{ }^{* * * *} p<.0001$ 


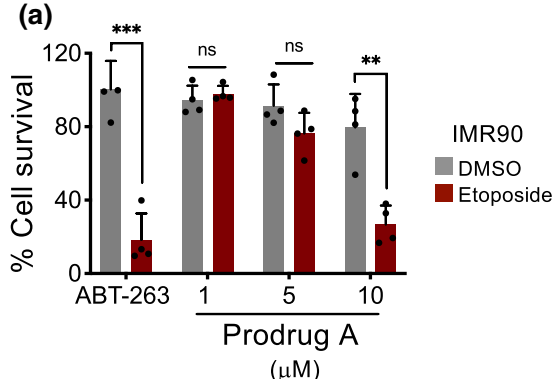

(c)

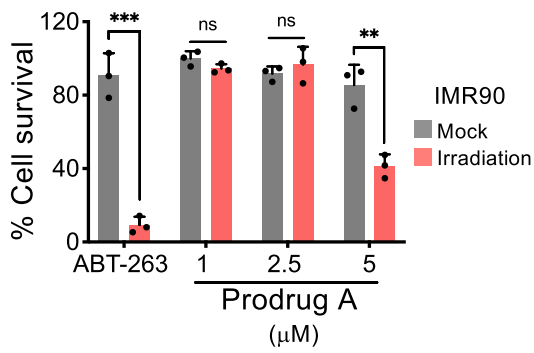

(e)

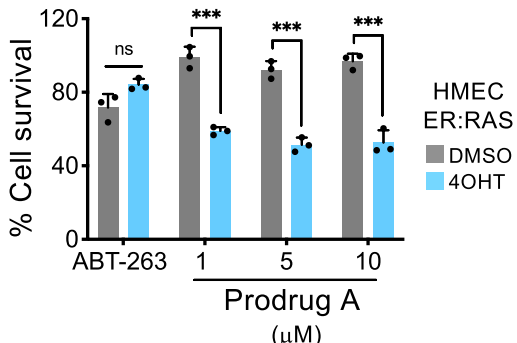

(b)

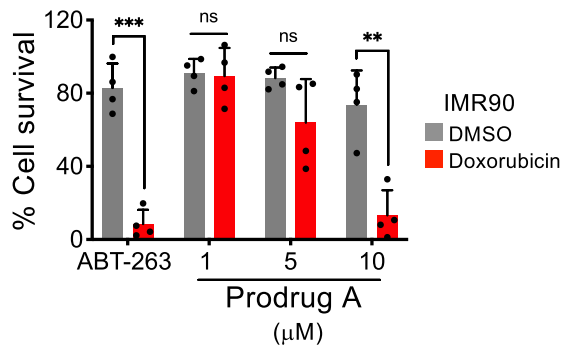

(d)

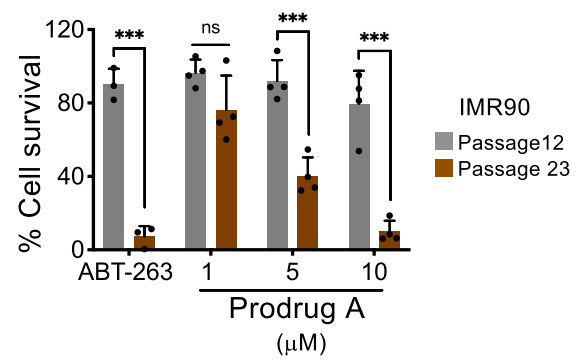

(f)
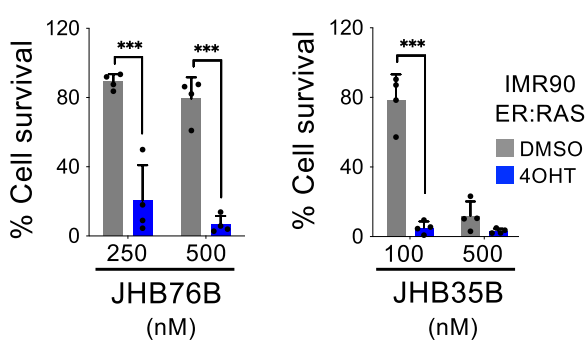

FIGURE 3 Galactose-modified duocarmycin prodrugs are broad-spectrum senolytics. (a-d) Quantification of cell survival after treatment with prodrug A in IMR90 undergoing different types of senescence. Senescence was induced by treatment with $50 \mu \mathrm{M}$ etoposide (a, $n=4), 0.5 \mu$ M doxorubicin (b, $n=4$ ) or $7.5 \mathrm{~Gy}$ irradiation (c, $n=3$ ). In (d), the effect of prodrug A on replicative senescence of IMR90 cells (passage 12 versus passage 22) $(n=4)$ was assessed. (e) Quantification of cell survival after treatment with prodrug A in HMEC ER:RAS, human mammary epithelial cells expressing hTERT that undergo senescence upon activation of ER:RAS by $4 \mathrm{OHT}$ treatment $(n=3)$. (f) Quantification of cell survival after treatment with two other galactose-modified duocarmycin derivatives, JHB76B and JHB35B, in the context of oncogene-induced senescence in IMR90 ER:RAS $(n=4)$. An extended version of this figure including additional drug concentrations is shown in Figure S2. All statistical significances were calculated using unpaired Student's $t$ tests. All error bars represent mean $\pm S D ; n$ represents independent experiments; ns, not significant; ${ }^{*} p<.05 ;{ }^{* *} p<.01 ;{ }^{* * *} p<.001$

Figure S2), linking the senolytic selectivity of prodrug A with the SA$\beta$-galactosidase activity.

The increase in $\beta$-galactosidase observed on senescent cells is due to an increase in lysosomal mass (Kurz et al., 2000) resulting in higher activity of the lysosomal $\beta$-galactosidase (encoded by GLB1) (Lee et al., 2006). To further prove that the senolytic activity of GMD prodrugs is dependent on SA- $\beta$-galactosidase, we took advantage of three independent shRNAs to knock down GLB1 (Figure 2d). Knock-down of GLB1 in IMR90 ER:RAS cells resulted in decreased SA- $\beta$-galactosidase activity, but it did not impact the growth arrest or the induction of $\mathrm{p} 16^{\mathrm{INK} 4 \mathrm{a}}$ observed during OIS (Figure 2e,f and Figure S3a,b). Taking advantage of these cells, we observed that GLB1 knock-down did not affect the senolytic potential of ABT-263 but ablated the ability of prodrug $A$ to selectively kill senescent cells (Figure $2 \mathrm{~g}$ and Figure S3c). In summary, our data suggest that GMD prodrugs trigger apoptosis of senescent cells in a GLB1-dependent manner.

\section{3 | Galactose-modified duocarmycin prodrugs are broad-spectrum senolytics}

To understand the extent to which GMD prodrugs behave as senolytics, we assessed the effect that prodrug A has on several types of senescent cells. To this end, we took advantage of IMR90 cells and induced senescence by etoposide or doxorubicin treatment, irradiation, or serial passage (Figure S4a-d). In all those instances, treatment with prodrug $A$ resulted in the selective elimination of senescent cells (Figure 3a-d). Moreover, to evaluate whether the senolytic effects of prodrug A were restricted to IMR90 cells or also observed in other cell types, we took advantage of human mammary epithelial cells able to undergo OIS upon Ras activation (HMEC ER:RAS, Figure S4e). Prodrug A was also able to selectively kill HMEC senescent cells (Figure 3e), suggesting that its senolytic effects were not cell type restricted. Finally, we wanted to examine whether the senolytic properties were specific of prodrug $A$, or the 
general concept (conversion of other cytotoxic drugs in galactosemodified prodrugs) was wider. To this end, we took advantage of two previously described GMD prodrugs, JHB76B and JHB35B (Tietze et al., 2009, 2010). Both drugs were also effective in selectively eliminating senescent cells (Figure $3 \mathrm{f}$ and Figure S5), suggesting that the generation of galactose-modified prodrugs might be a general route to design senolytic compounds.

\section{4 | Prodrug A exerts a bystander effect}

The above experiments suggest that senescent cells preferentially convert GMD prodrugs into their active duocarmycin derivatives. Since duocarmycins have strong cytostatic properties (Boger \& Johnson, 1995), we wonder whether the conversion of GMD prodrugs into their active compounds could result in the bystander killing of normal cells. To assess this, we took advantage of cocultures of normal and senescent cells expressing different fluorescent proteins (Figure 4a). We then treated the cocultures with prodrug $A$ and observed that while lower concentrations killed a subset of senescent cells without affecting to the normal cells, at higher concentrations, a subset of the normal cells also died (Figure $4 b, c$ ). Interestingly, a higher percentage of senescent cells survived in cocultures (Figure 4c) that in monocultures (Figure 1b) treated with $5 \mu \mathrm{M}$ prodrug $\mathrm{A}$, further suggesting the existence of a bystander effect.

\section{5 | Prodrug A eliminates senescent cells in vivo}

Chemotherapy and radiotherapy are amongst the most common anticancer treatments. Irradiation, chemotherapy and even some targeted anticancer drugs, all induce senescence (Schmitt et al., 2002; Wang et al., 2017). Although induction of tumour senescence explains the anticancer properties of these treatments, the generation of bystander senescent cells is responsible for their side effects (Demaria et al., 2017). To assess whether prodrug A could eliminate these senescent cells, we first irradiated mice and upon a latency period to allow for the accumulation of senescent cells, treated them with prodrug A, ABT263 or vehicle (Figure 5a). Treatment with prodrug $A$ or $A B T-263$ resulted in a reduced presence of senescent cells in lung as assessed using SA- $\beta$-galactosidase activity (Figure $5 b, c$ ). Furthermore, a similar trend was observed when we assessed the expression of $C d k n 1 a$ (that encodes for $\mathrm{p} 21^{\mathrm{Cip} 1}$ ) or the SASP components 116 and $\mathrm{Cxcl1}$ (Figure $5 \mathrm{~d}$ ).

\section{6 | Galactose-modified prodrugs eliminate preneoplastic senescent cells}

OIS is primarily considered as a tumour suppressive mechanism (Collado et al., 2005), but senescent cells present in the tumour microenvironment can also drive tumour progression (Gonzalez-Meljem, (a)

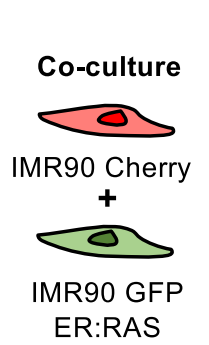

(b)

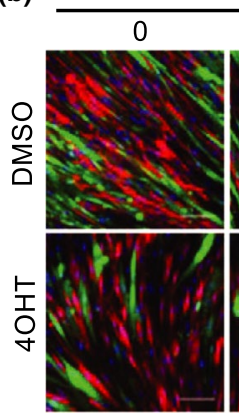

Prodrug $\mathrm{A}(\mu \mathrm{M})$

2.5

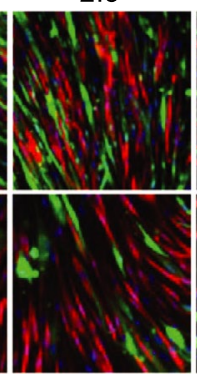

5

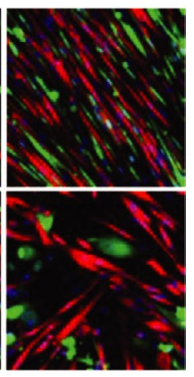

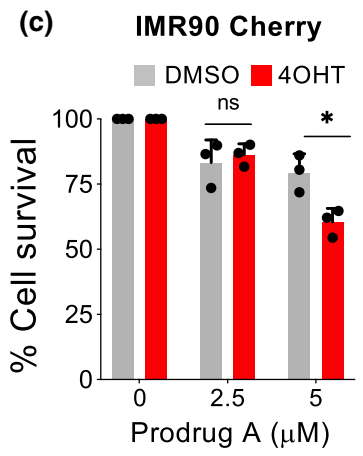

IMR90 GFP ER:RAS DMSO $\square$ 4OHT

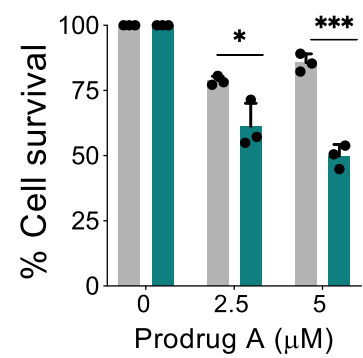

FIGURE 4 Bystander effect caused by prodrug $A$ in cocultures of normal and senescent cells. (a) Experimental set-up. (b) Representative pictures of the co-culture experiment of IMR90 ER:RAS (GFP positive) with wild-type IMR90 (RFP positive) cells. (c) Quantification of cell survival for each population of the coculture experiment after treatment with different concentrations of prodrug A or vehicle $(n=3)$. Statistical significance was calculated using unpaired Student's $t$ tests. All error bars represent mean \pm s.d; $n$ represents independent experiments; ns, not significant; ${ }^{*} p<.05$; ${ }^{* *} p<.01 ;{ }^{* * *} p<.001$

Apps, Fraser, \& Martinez-Barbera, 2018). We have previously demonstrated in mouse models of adamantinomatous craniopharyngioma (ACP), a pituitary paediatric tumour, that clusters of cells that accumulate nucleo-cytoplasmic $\beta$-catenin are senescent and drive tumour progression in a paracrine manner (Gonzalez-Meljem et al., 2017). Indeed, $\beta$-catenin-positive cells are ki67 negative, express $\mathrm{p} 21^{\mathrm{Cip} 1}$ and high levels of the lysosomal $\beta$-galactosidase GLB1 (Figure S6a) (Gonzalez-Meljem et al., 2017). To understand if GMD prodrugs could eliminate these pro-tumourigenic senescent clusters, we used the $\mathrm{Hes \times 1}{ }^{\mathrm{Cre} /+} ; \mathrm{Ctnn} b 1^{\text {lox(ex } 3) /+}$ ACP mouse model. We have used this system before to assess the senolytic properties of cardiac glycosides (Guerrero et al., 2019). Tumoural cluster-containing embryonic pituitaries were cultured ex vivo with vehicle or prodrug A (Figure 6a). Treatment with prodrug A preferentially eliminated the $\beta$-catenin-accumulating senescent cell clusters, without affecting other cell types in the pituitary such as synaptophysin + cells (Figure 6b-d). Co-staining with an antibody recognizing cleaved caspase 3 showed that prodrug A predominantly induced apoptosis of senescent cluster cells (Figure 6e and Figure S6b). The above results suggest that GMD prodrugs could be also used to eliminate preneoplastic senescent cells. 
FIGURE 5 Prodrug A reduces the numbers of senescent cells accumulating after whole-body irradiation. (a)

Experimental design of the wholebody irradiation-induced senescence experiment. Mice ( $n=4 / 5$ per group) were irradiated with 6 Gray to induce senescence. Two months later, mice were treated with vehicle, prodrug $A$ (JHB75B) or ABT-263 for 4 consecutive days, before being culled for analysis. (b, c) Representative pictures of lung cryosections (b) and quantification of the lung area positive for SA- $\beta$-Gal staining (c). (d) Expression levels of Cdkn1a, Il6 and Cxcl1 in lungs of nonirradiated mice or irradiated mice treated with prodrug $A$, ABT-263 or vehicle. Statistical significance was calculated using unpaired Student's $t$ test. Data represent mean $\pm S D ; n$ represents number of mice; ns, not significant; ${ }^{*} p<.05 ;{ }^{* *} p<.01$ (a)

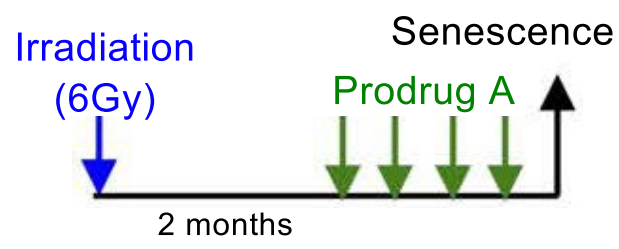

(b)
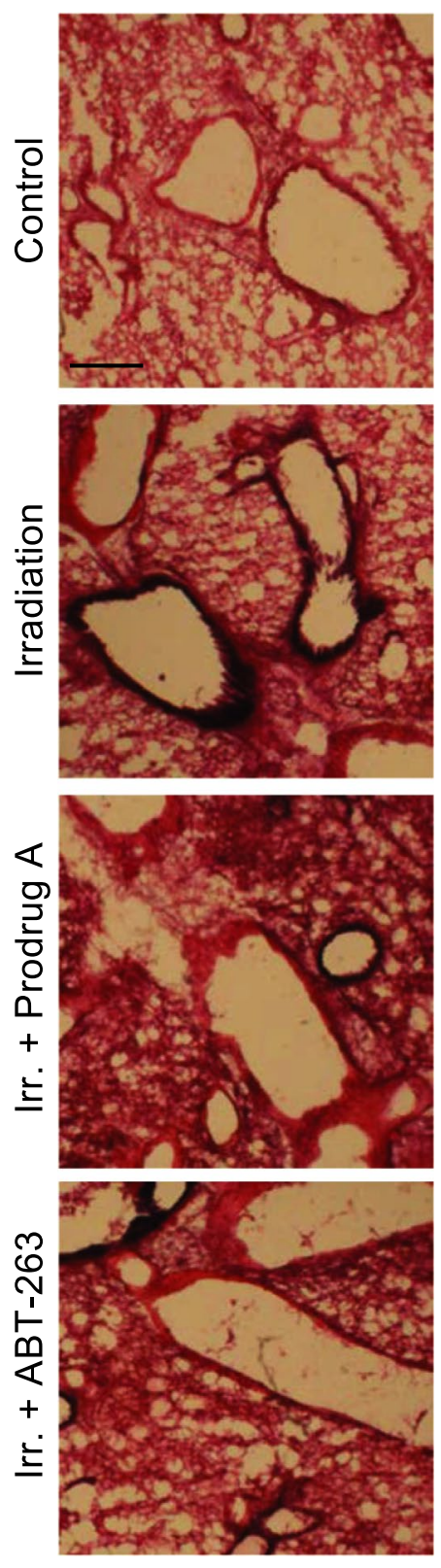

SA- $\beta-$ Gal
SA- $\beta-$ Gal

(c)

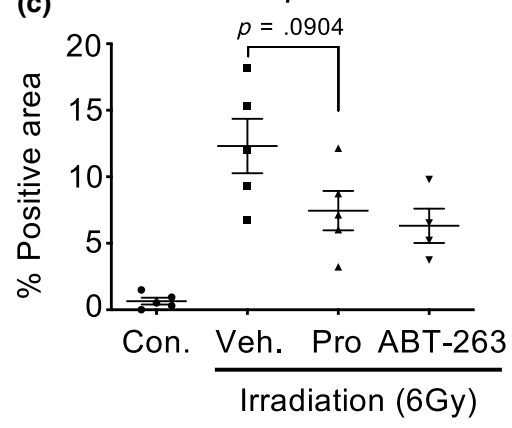

(d)

\section{Cdkn1a}
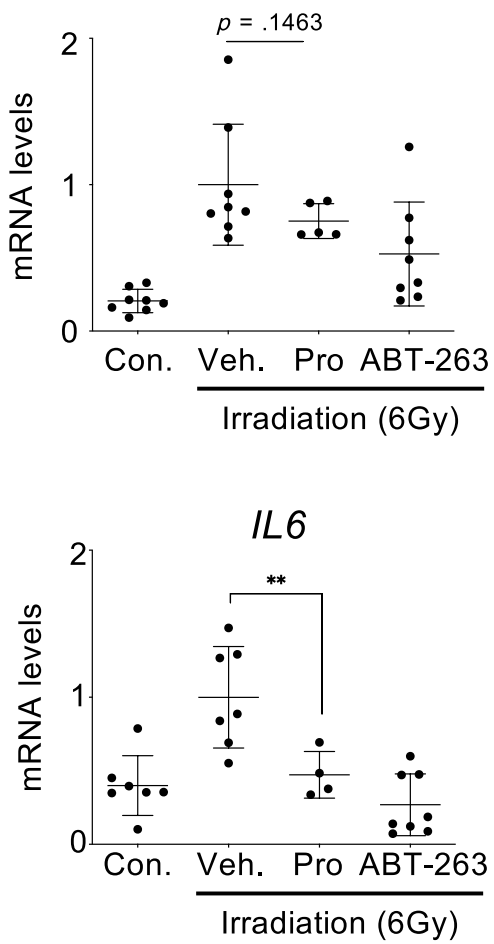

\section{Cxcl1}

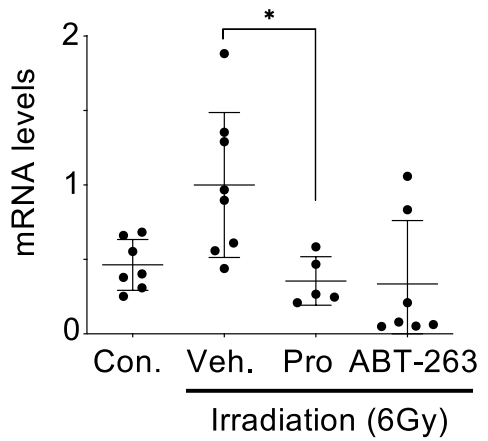

\section{3 | DISCUSSION}

Recently, the use of genetic mouse models in which senescent cells can be ablated has served to unveil important roles for senescence in health, disease and ageing (Baker et al., 2011, 2016; Demaria et al.,
2014). Consequently, drugs have been identified that are able to phenocopy the effects of selectively eliminating senescent cells. Several of these so-called senolytic drugs have been discovered, with Bcl2 family inhibitors such as ABT-263 (navitoclax) and ABT-737 (Chen et al., 2015; Yosef et al., 2016; Zhu et al., 2016) being the prototypical examples. 
Here, we add galactose-modified duocarmycin (GMD) prodrugs as a new class of senolytic agents. These GMD prodrugs are converted to their corresponding duocarmycin drugs in a manner dependent on processing by $\beta$-galactosidase. Since senescent cells display elevated levels of lysosomal $\beta$-galactosidase (encoded by
GLB1), GMD selectively affects senescent cells. In this manuscript, we present evidence showing that GMD prodrugs can eliminate multiple types of senescent cells, what is consistent with SA- $\beta$ galactosidase being a universal marker of senescence. Our preliminary results suggest that GMD prodrugs could also be capable of (a)

Hes $\times 1^{\mathrm{Cre} /+}$

Ctnnb $1^{\text {lox }(\mathrm{ex} 3) /+}$

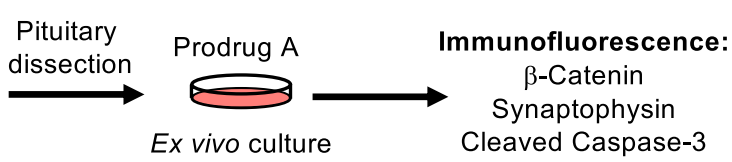

Ex vivo culture
Cleaved Caspase-3

(b)

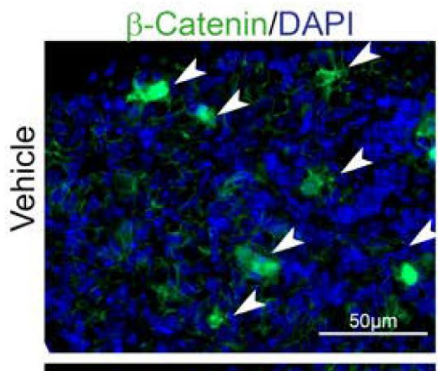

Synaptophysin/DAPI

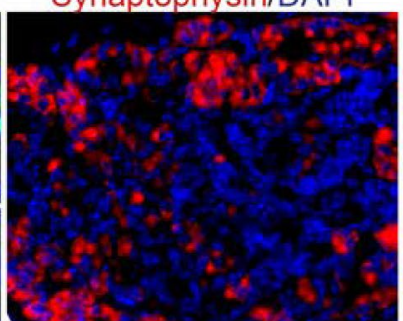

Synapto/3-Cat/DAPI
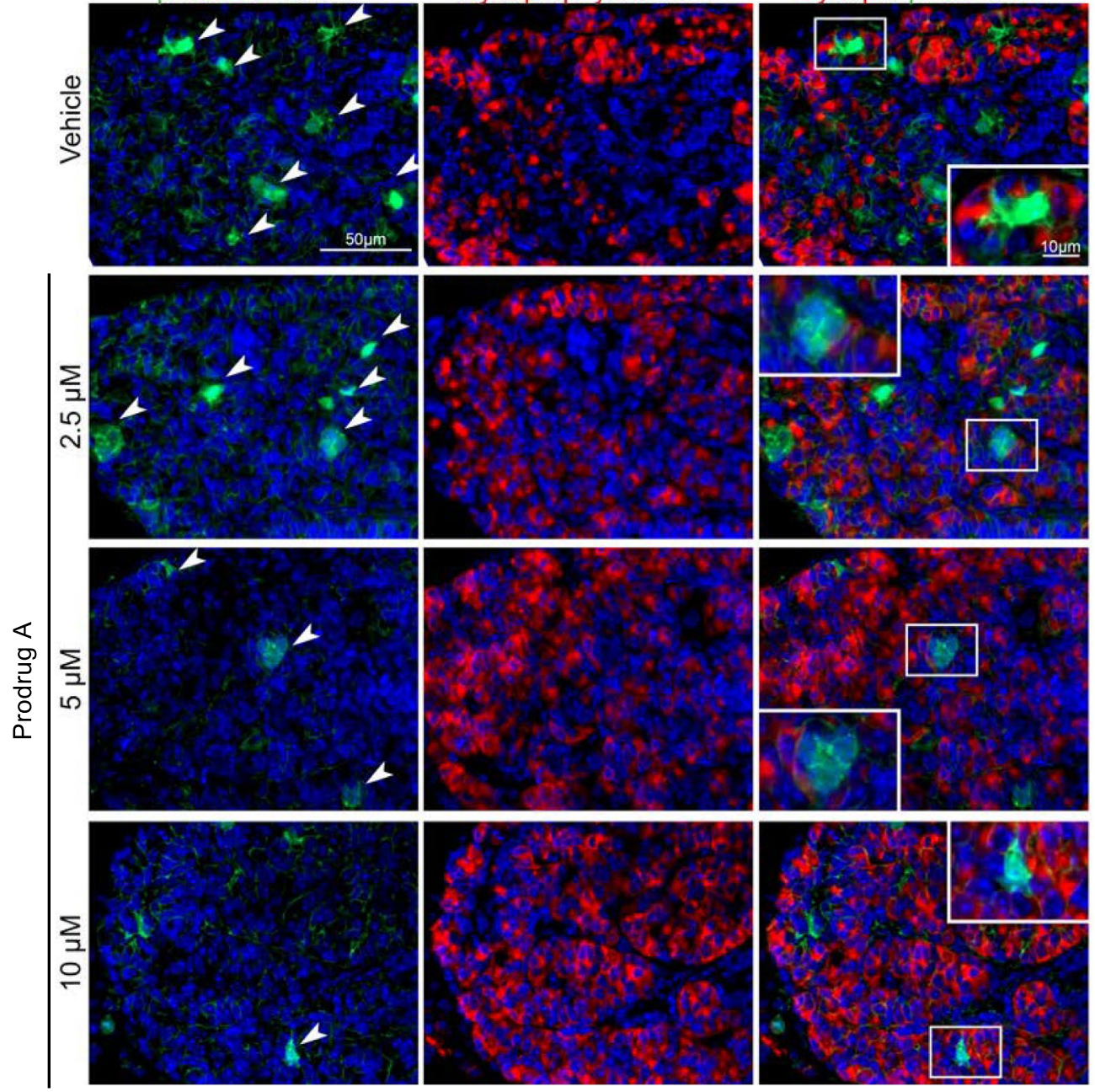

(c)

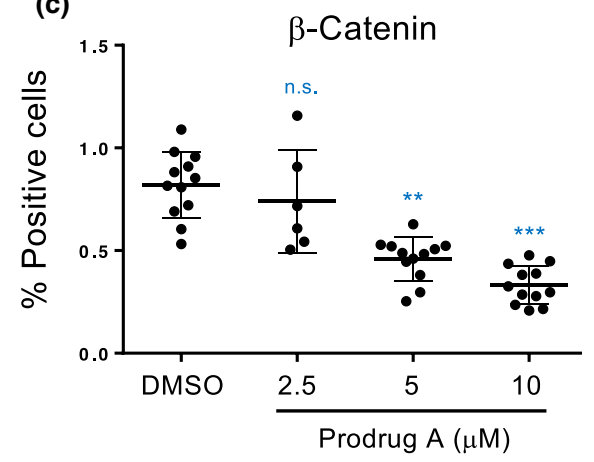

(d)

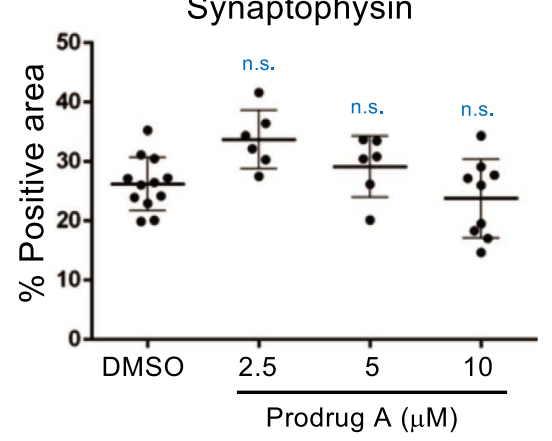

(e)

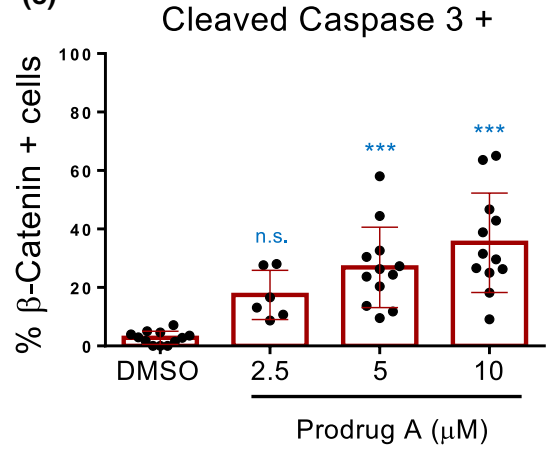


FIGURE 6 Galactose-modified duocarmycin prodrug eliminates preneoplastic senescent lesions. (a) Experimental design for the senolytic experiment in the Hesx $1^{\text {Cre/+ }} ;$ Ctnnb $1^{\text {lox(ex3)/+ }}$ mouse model of adamantinomatous craniopharyngioma (ACP). Tumoural pituitaries from $18.5 \mathrm{dpc} \mathrm{Hes \times 1} 1^{\mathrm{Cre} /+} ; \mathrm{Ctnnb} 1^{\text {lox(ex3)/+ }}$ embryos were cultured in the presence of prodrug $\mathrm{A}$ at the indicated concentrations or vehicle (DMSO) and processed for analysis after $72 \mathrm{hr}$. (b) Immunofluorescence staining against $\beta$-catenin (green) and synaptophysin (red) is shown. Synaptophysin is a marker of the normal hormone-producing cells in the pituitary gland. Scale bar, 50 $\mu \mathrm{m}$. (c) Quantification of $\beta$-cateninaccumulating cells after treatment with different concentrations of prodrug A or vehicle $(n=6-12)$. (d) Quantification of synaptophysinpositive cells after treatment with different concentrations of prodrug A or vehicle $(n=6-12)$. (e) Quantification of $\beta$-catenin-accumulating cells positive for cleaved caspase-3 after treatment with different concentrations of prodrug A or vehicle $(n=6-12)$. All statistical significances were calculated using nonparametric ANOVA with Dunn's post hoc comparison. All error bars represent mean $\pm S D ; n$ represents number of pituitaries; ns, not significant; ${ }^{*} p<.05 ;{ }^{* *} p<.01 ;{ }^{* * *} p<.001$

eliminating bystander senescent cells arising from anticancer therapies and preneoplastic senescent cells in mouse models. This needs further investigation.

Given the promise that senolytics present for the treatment of age-related disease, and their associated benefits over healthspan and lifespan, we believe that this study provides the basis to specifically assess the potential benefits of GMD on ageing.

Previously, the potential to harness the elevated $\beta$-galactosidase activity of senescent cells has been exploited with galacto-oligosaccharide encapsulated nanoparticles (GalNP) (Agostini et al., 2012). Combination of GalNP with different cargoes offers flexibility to image or eliminate senescent cells (Munoz-Espin et al., 2018). However, this flexibility comes to the expense of having to use a modular system, comprised of both the GaINP and the cargo. Here, we propose the use of galactose-modified prodrugs in which a single molecule (the prodrug) is sufficient to target senescent cells taking advantage of their elevated $\beta$-galactosidase activity. While we show that duocarmycin derivatives behave as senolytic agents, this approach could be generalized to galactose-modified prodrugs derived of other cytotoxic agents.

In summary, we have described that galactose-modified duocarmycin prodrugs are a new class of broad-spectrum senolytic agents. We have characterized their ability to eliminate different types of senescent cells in culture and carried out preliminary experiments in vivo. Given the increasing list of diseases that are associated with senescence, galactose-modified duocarmycin prodrugs have the potential to be used in the context of anticancer therapies and to treat different age-related diseases. The present study should provide the basis for investigating the potential of galactose-modified prodrugs as senolytics to treat senescence-associated diseases.

\section{4 | EXPERIMENTAL PROCEDURES}

\section{1 | Drugs}

The following compounds were used in this study: ABT-263 (Selleckchem, S1001), Etoposide (Sigma-Aldrich, E1383), Q-VD-OPh hydrate (Sigma-Aldrich, SML0063), 4-hydroxy-tamoxifen (SigmaAldrich, H7904), Doxorubicin hydrochloride (Cayman chemical, 15007). Galactose-modified prodrugs (JHB75B, referred as prodrug $A$ in the manuscript; JHB35B; JHB76B) and seco-Duocarmycin analog dimer (JHB71A) were provided by Prof. Dr. L. F. Tietze. All drugs were reconstituted in DMSO unless otherwise stated.

\subsection{Antibodies}

The following primary antibodies were used in this study: mouse monoclonal anti-BrdU (3D4; BD Biosciences, 555627), mouse monoclonal anti-p16 ${ }^{\text {INK4a }}$ (JC-8; from CRUK), rabbit polyclonal anti- $\beta$-Catenin (Thermo Fisher Scientific, RB-9035-P1), mouse polyclonal anti-p21 (BD Biosciences, 556,431), mouse monoclonal anti-Synaptophysin (27G12; Leica, NCL-L-SYNAP-299), rabbit monoclonal anticleaved caspase 3 (Asp175; 5A1E; Cell Signalling Technology, 9664), rabbit monoclonal Ki67 (Abcam, ab16667), mouse monoclonal pSer32/36-ІкB $\alpha$ (Cell Signalling Technology, 9246), mouse monoclonal pT2609-DNA PKcs (Abcam, 18,356), rabbit polyclonal GLB1 (Proteintech, 15518-1AP). We used the following secondary antibodies: goat anti-mouse IgG $(H+L$, Alexa Fluor 488 conjugated, Thermo Fisher Scientific, A11029), goat anti-mouse IgG $(\mathrm{H}+\mathrm{L}$, Alexa Fluor 594 conjugated, Thermo Fisher Scientific, A11032), goat anti-rabbit IgG $(\mathrm{H}+\mathrm{L}$, Alexa Fluor 594 conjugated, Thermo Fisher Scientific, A11037) and goat anti-rabbit IgG-HRP (Santa Cruz, sc-2004).

\subsection{Cell lines}

IMR90 cells were obtained from ATCC. IMR90 ER:RAS were generated by retroviral infection of IMR90 cells and have been described elsewhere (Banito et al., 2009; Barradas et al., 2009). IMR90 were cultured in DMEM (Gibco) supplemented with $10 \%$ foetal bovine serum (Sigma) and 1\% antibiotic-antimycotic solution (Gibco). HMEC were cultured in Medium 171 (Gibco) supplemented with MEGS (Gibco), 10\% foetal bovine serum (Sigma) and 1\% antibiotic-antimycotic solution (Gibco). To induce OIS, IMR90 ER:RAS and HMEC ER:RAS were treated with $100 \mathrm{nM}$ 4-hydroxy-tamoxifen $(4 \mathrm{OHT}$, Sigma) for 6 days. To induce chemotherapy-induced senescence, IMR90 cells were treated with $0.5 \mu \mathrm{M}$ Doxorubicin (Sigma) for $24 \mathrm{hr}$, or with $50 \mu \mathrm{M}$ Etoposide (Sigma) for $48 \mathrm{hr}$ (DMSO-treatment was used as a control). To induce senescence by ionizing radiation, IMR90 were $\gamma$-irradiated (7.5 Gy). For therapy-induced senescence experiments (chemotherapy and irradiation), senolytic drugs were added 7 days after the induction of senescence. 


\section{4 | Vector construction}

pGIPZ-based shRNA targeting GLB1 (V3LHS_361778, V3LHS_361779, V2LHS_232389) were obtained from MRC LMS Genomics core facility.

To generate IMR90 ER:RAS-expressing shRNAs against GLB1, lentiviral infections were carried out as described before (Aarts et al., 2017). Briefly, HEK293T cells were transfected with the lentiviral and packaging vectors using PEI (PEI 2500, Polysciences). Two days after transfection, HEK293T viral supernatants were collected, filtered $(0.45 \mu \mathrm{M})$, diluted $1 / 4$, supplemented with $4 \mu \mathrm{g} / \mathrm{ml}$ polybrene and added to IMR90 ER:RAS cells plated the day before at a density of 1 million cells per $10-\mathrm{cm}$ dish. Four hours later, lentivirus-containing media was replaced with fresh media. Three days after infection, cells were passaged and cultured for three days in the presence of $1 \mu \mathrm{g} / \mathrm{ml}$ puromycin (InvivoGen) to select for infected cells.

\section{5 | BrdU incorporation}

BrdU incorporation assays were performed as previously described (Georgilis et al., 2018). Briefly, for BrdU incorporation assays, the cells were incubated with $10 \mu \mathrm{M}$ BrdU for 16-18 hr before being fixed with $4 \%$ PFA (w/v). BrdU incorporation was assessed by Immunofluorescence and High Content Analysis microscopy.

\subsection{Immunofluorescence staining of cells}

Cells were grown in 96-well plates, fixed with $4 \%$ PFA (w/v) and stained as previously described (Georgilis et al., 2018).

\section{7 | Cytochemical SA- $\beta$-galactosidase assay}

Cells were grown on 6-well plates, fixed with $0.5 \%$ glutaraldehyde $(\mathrm{w} / \mathrm{v})$ (Sigma) in PBS for 10-15 min, washed with $1 \mathrm{mM} \mathrm{MgCl}_{2} / \mathrm{PBS}$ $(\mathrm{pH} 6.0)$ and then incubated with $X-G a l$ staining solution (1 mg/ $\mathrm{ml} \mathrm{X-Gal,} \mathrm{Thermo} \mathrm{Fisher} \mathrm{Scientific,} 5 \mathrm{mM} \mathrm{K}_{3}\left[\mathrm{Fe}(\mathrm{CN})_{6}\right]$ and $5 \mathrm{mM}$ $\mathrm{K}_{4}\left[\mathrm{Fe}(\mathrm{CN})_{6}\right]$ for $8 \mathrm{hr}$ at $37^{\circ} \mathrm{C}$. Bright field images of cells were taken using the DP20 digital camera attached to the Olympus CKX41 inverted light microscope. The percentage of SA- $\beta$-Gal-positive cells was estimated by counting at least 100 cells per replicate sample facilitated by the "point picker" tool of ImageJ software (NIH).

For SA- $\beta$-galactosidase staining in tissues, frozen sections $(6 \mu \mathrm{m})$ were fixed in ice-cold $0.5 \%$ glutaraldehyde $(\mathrm{w} / \mathrm{v})$ solution for $15 \mathrm{~min}$, washed with $1 \mathrm{mM} \mathrm{MgCl}_{2} / \mathrm{PBS}$ ( $\mathrm{pH}$ 6.0) for $5 \mathrm{~min}$ and then incubated with $\mathrm{X}$-Gal staining solution for $16-18 \mathrm{hr}$ at $37^{\circ} \mathrm{C}$ as previously described (Georgilis et al., 2018). After the staining, the slides were counterstained with eosin, dehydrated, mounted and analysed by phase-contrast microscopy. SA- $\beta$-Gal tissue staining was quantified using Image J software (NIH) by measuring the percentage of stained area in each section and multiplying it by its mean intensity value as described before (Tordella et al., 2016). To exclude the luminal spaces in the lung sections, the percentage of SA- $\beta$-Gal-positive area was divided by the total lung area, as determined by eosin-positive area using ImageJ (NIH).

\section{8 | Fluorescent SA- $\beta$-galactosidase assay}

Cells were grown on 96-well plates. After 8 days, cells were treated with $100 \mu \mathrm{M}$ DDAOG (D-6488, Thermo Fisher Scientific) $2 \mathrm{hr}$ before fixation with $4 \%$ PFA for $15 \mathrm{~min}$. Nuclei were stained with DAPI $(1 \mu \mathrm{g} / \mathrm{ml})$ for $15 \mathrm{~min}$ and images acquired the same day using a IN Cell Analyzer 2000 (GE Healthcare).

\subsection{Determining senolytic activity}

For oncogene-induced senescence experiments, IMR90 ER:RAS or HMEC ER:RAS cells were plated in 96-well dishes and induced to undergo senescence by treating them with $100 \mathrm{nM} 4 \mathrm{OHT}$ for 6 days. From that point, cells were kept under serum-starvation conditions (0.5\% FBS) and $1 \mu \mathrm{M}$ ABT-263 or different concentrations of the galactose-modified prodrugs were added. In parallel, the same treatments were carried out in IMR90 ER:RAS or HMEC ER:RAS cells treated with DMSO $(-4 \mathrm{OHT})$. These cells do not undergo senescence. Cells were fixed at day 9 after $4 \mathrm{OHT}$ induction and stained with DAPI $(1 \mu \mathrm{g} / \mathrm{ml})$ for $15 \mathrm{~min}$ to assess cell numbers using automated microscopy. Different models of senescence were used to test the senolytic activity of galactose-modified prodrugs in cell culture in a similar fashion. Briefly, for therapy-induced senescence IMR90 cells were treated with $50 \mu \mathrm{M}$ etoposide (48 hr), $0.5 \mu \mathrm{M}$ doxorubicin ( $24 \mathrm{hr}$ ) or left untreated, and then kept in drugfree complete media until day 7 , when the senolytics were added. Cells were fixed at day 10 after senescence induction. In all senescence types tested, 3-day course of senolytics was applied. The percentage of cell survival was calculated dividing the number of cells after drug treatment by the number of cells treated with vehicle.

\subsection{0 | Co-culture experiment}

IMR90 ER:RAS (GFP positive) were plated in 96-well plates and treated with DMSO or $4 \mathrm{OHT}$ for 6 days. Then, IMR90 (RFP positive) were added on top. On day 7 , the cocultures were treated with different concentrations of the prodrug $A$ or vehicle and cell survival for each population was calculated.

\subsection{1 | High content analysis (HCA)}

IF imaging was carried out using the automated high-throughput fluorescent microscope IN Cell Analyzer 2000 (GE Healthcare) 
with a $20 \times$ objective. Multiple fields within a well were acquired in order to include a minimum of 1,000 cells per sample-well. HCA of the images was processed using the INCell Investigator 2.7 .3 software as described previously (Herranz et al., 2015). Briefly, DAPI served as a nuclear mask hence allowed for the segmentation of cells with a Top-Hat method. To detect cytoplasmic staining in cultured cells, a collar of 7-9 $\mu \mathrm{m}$ around DAPI was applied. In samples of cultured cells, a threshold for positive cells was assigned above the average intensity of unstained or negative control sample unless otherwise specified.

\subsection{2 | IncuCyte analysis}

IMR90 ER:RAS cells were plated in 96-well dishes and induced to undergo senescence as previously described. Different concentrations of galactose-modified prodrug were added as normally. Cell culture media was supplemented with IncuCyte NucLight Rapid Red Reagent for cell labelling (Essen Bioscience) and IncuCyte Caspase-3/7 reagent for apoptosis (Essen Bioscience). Four images per well were collected every 2 hr for 3 days using a 10x objective.

\subsection{3 | Gene expression analysis}

Total RNA was extracted using Trizol reagent (Invitrogen) and the RNeasy isolation kit (Qiagen). cDNA was generated using random hexamers and SuperScript II reverse transcriptase (Invitrogen). Quantitative real-time PCR was performed using SYBR Green PCR master mix (Applied Biosystems) in a CFX96 real-time PCR detection system (Bio-Rad). Rps14 expression was used for normalization. Mouse primer pairs are:

Cdkn1a: CAGATCCACAGCGATATCCA, ACGGGACCGAAGAGA CAAC.

Cxcl1: CTGGGATTCACCTCAAGAACATC, CAGGGTCAAGGCAA GCCTC.

CD68: AGGACCGCTTATAGCCCAAG, GGATGGCAGGAGAGTA ACGG

Il6: TGATTGTATGAACAACGATGATGC, GGACTCTGGCTTTGT CTTTCTTGT.

Rps14: GACCAAGACCCCTGGACCT, CCCCTTTTCTTCGAGT GCTA.

\subsection{Mouse models and drug treatments}

For induction of senescence, $\mathrm{C} 57 \mathrm{BL} / 6 \mathrm{~J}$ mice at 8-12 weeks of age were exposed to a sublethal dose ( $6 \mathrm{~Gy}$ ) of total body irradiation. Eight weeks after, mice were injected with 50 nmols of prodrug $A$ (i.v.) or vehicle for four consecutive days. Mice were killed $24 \mathrm{hr}$ after the last injection. Mice lungs were harvested for RNA extraction, paraffin embedded for immunohistology, or frozen in OCT/Sucrose 15\% (1:1) solution for cryosectioning and SA- $\beta$-gal stains. The mice used for all experiments were randomly assigned to control or treatment groups. Both sexes were used throughout the study.

For in vivo treatment, ABT-263 was prepared in ethanol:polyethylene glycol 400:Phosal 50 PG at 10:30:60 as previously described (Chang et al., 2016). Mice were gavaged with vehicle (ethanol:polyethylene glycol 400:Phosal 50 PG) or ABT-263 (50 mg/kg).

All mouse procedures were performed under licence, following UK Home Office Animals (Scientific Procedures) Act 1986 and local institutional guidelines (UCL or Imperial College ethical review committees).

\subsection{Ex vivo culture of mouse pituitaries}

Neoplastic pituitaries from $18.5 \mathrm{dpc} \mathrm{Hes} 1^{\mathrm{Cre} /+} ; \mathrm{Ctnn} 1^{\text {lox(ex3)/+ }} \mathrm{em}-$ bryos were dissected and placed on top of $0.2 \mu \mathrm{M}$ Whatman filters (SLS) in 24-well plates containing $500 \mu \mathrm{l}$ of media (DMEM-F12, Gibco, 1\% Pen/Strep, Sigma and 1\% FBS, PAA) supplemented with either prodrug $A$ or vehicle (DMSO). Media was changed every $24 \mathrm{~h}$, and pituitaries were processed for analysis after $72 \mathrm{hr}$. Immunofluorescence staining was performed as previously described (Gonzalez-Meljem et al., 2017). The proportion of $\beta$-cateninaccumulating and p21-positive cells was calculated as an index out of the total DAPI-stained nuclei. The proportion of $\beta$-cateninaccumulating, cleaved caspase- 3 and p21-positive cells was calculated as an index out of the total DAPI-stained nuclei. Over 300,000 DAPI nuclei were counted from ten histological sections per sample, in a total of twelve neoplastic pituitaries.

\subsection{6 | Statistical analysis}

GraphPad Prism 7.0 was used for statistical analysis. Two-tailed Student's $t$ tests were used to estimate statistically significant differences between two groups. Two-way ANOVA with Tukey's post hoc comparison was used for multiple comparisons. Values are presented as mean $\pm S D$ unless otherwise indicated. Asterisks $\left({ }^{*}\right)$ always indicate significant differences as follows: ns = not significant, ${ }^{*} p<.5,{ }^{* *} p<.01,{ }^{* * *} p<.001,{ }^{* * * *} p<.0001$

For in vivo studies, mice were randomly assigned to treatment groups. All replicates in this study represent different mice.

\section{ACKNOWLEDGMENTS}

We are grateful to members of J. Gil's laboratory for reagents, comments and other contributions to this project. Core support from Medical Research Council (MC-A652-5PZO0 and MC_ U120085810) funded this research in J. Gil's laboratory. D.J.W. was funded by Wellcome Trust (Strategic Award 098565) and core support from Medical Research Council (MC-A654-5QB40). J.P.M.-B. was funded by the Brain Tumour Charity (SIGNAL and EVEREST), Children with Cancer UK, CRUK, Great Ormond Street Hospital Charity and NIHR Biomedical Research Centre at 
University College London Hospitals NHS Foundation Trust and University College London.

\section{CONFLICT OF INTEREST}

J.G. owns equity and has acted as a consultant for Unity Biotechnology and Geras Bio. J.G., A.G. and N.H. are named inventors in an MRC patent related to senolytic therapies (not related to the work presented here).

\section{AUTHORS' CONTRIBUTION}

A.G. designed performed and analysed the cell culture experiments and wrote the first draft of the manuscript. R.G. designed performed and analysed the experiments with the ACP model. N.H. designed, performed and analysed the in vivo experiments. L.F.T. designed and synthetized the duocarmycin derivatives and secured funding. A.U., J.P. M.-B. and D.J.W. designed the in vivo experiments and secured funding. J.G. conceived and designed the project, secured funding and wrote the manuscript, with all authors providing feedback.

\section{DATA AVAILABILITY STATEMENT}

The data that support the findings of this study are available from the corresponding author upon reasonable request.

\section{ORCID}

Jesús Gil iD https://orcid.org/0000-0002-4303-6260

\section{REFERENCES}

Aarts, M., Georgilis, A., Beniazza, M., Beolchi, P., Banito, A., Carroll, T., ... Gil, J. (2017). Coupling shRNA screens with single-cell RNA-seq identifies a dual role for mTOR in reprogramming-induced senescence. Genes \& Development, 31, 2085-2098.

Acosta, J. C., Banito, A., Wuestefeld, T., Georgilis, A., Janich, P., Morton, J. P., ... Gil, J. (2013). A complex secretory program orchestrated by the inflammasome controls paracrine senescence. Nature Cell Biology, 15, 978-990.

Acosta, J. C., O'Loghlen, A., Banito, A., Guijarro, M. V., Augert, A., Raguz, S., ... Gil, J. (2008). Chemokine signaling via the CXCR2 receptor reinforces senescence. Cell, 133, 1006-1018.

Agostini, A., Mondragon, L., Bernardos, A., Martinez-Manez, R., Marcos, M. D., Sancenon, F., ... Murguia, J. R. (2012). Targeted cargo delivery in senescent cells using capped mesoporous silica nanoparticles. Angewandte Chemie International Edition, 51, 10556-10560.

Baar, M. P., Brandt, R. M. C., Putavet, D. A., Klein, J. D. D., Derks, K. W. J., Bourgeois, B. R. M., ... de Keizer, P. L. J. (2017). Targeted Apoptosis of Senescent Cells Restores Tissue Homeostasis in Response to Chemotoxicity and Aging. Cell, 169(132-147), e116.

Bagshawe, K. D. (2006). Antibody-directed enzyme prodrug therapy (ADEPT) for cancer. Expert Review of Anticancer Therapy, 6, 1421-1431.

Baker, D. J., Childs, B. G., Durik, M., Wijers, M. E., Sieben, C. J., Zhong, J., ... van Deursen, J. M. (2016). Naturally occurring p16(Ink4a)-positive cells shorten healthy lifespan. Nature, 530, 184-189.

Baker, D. J., Wijshake, T., Tchkonia, T., LeBrasseur, N. K., Childs, B. G., van de Sluis, B., ... van Deursen, J. M. (2011). Clearance of p16Ink4a-positive senescent cells delays ageing-associated disorders. Nature, 479, 232-236.

Banito, A., Rashid, S. T., Acosta, J. C., Li, S., Pereira, C. F., Geti, I., ... Gil, J. (2009). Senescence impairs successful reprogramming to pluripotent stem cells. Genes \& Development, 23, 2134-2139.
Barradas, M., Anderton, E., Acosta, J. C., Li, S., Banito, A., RodriguezNiedenfuhr, M., ... Gil, J. (2009). Histone demethylase JMJD3 contributes to epigenetic control of INK4a/ARF by oncogenic RAS. Genes \& Development, 23, 1177-1182.

Boger, D. L., \& Johnson, D. S. (1995). CC-1065 and the duocarmycins: Unraveling the keys to a new class of naturally derived DNA alkylating agents. Proceedings of the National Academy of Sciences of the United States of America, 92, 3642-3649.

Boger, D. L., Johnson, D. S., \& Yun, W. Y. (1994). (+)- and Ent-(-)Duocarmycin Sa and (+)- and Ent-(-)-N-Boc-Dsa DNA Alkylation Properties - Alkylation Site Models That Accommodate the Offset at-Rich Adenine N3 Alkylation Selectivity of the Enantiomeric Agents. Journal of the American Chemical Society, 116, 1635-1656.

Chang, J., Wang, Y., Shao, L., Laberge, R. M., Demaria, M., Campisi, J., ... Zhou, D. (2016). Clearance of senescent cells by ABT263 rejuvenates aged hematopoietic stem cells in mice. Nature Medicine, 22, 78-83.

Chen, Q., Song, S., Wei, S., Liu, B., Honjo, S., Scott, A., ... Ajani, J. A. (2015). ABT-263 induces apoptosis and synergizes with chemotherapy by targeting stemness pathways in esophageal cancer. Oncotarget, 6, 25883-25896.

Childs, B. G., Baker, D. J., Wijshake, T., Conover, C. A., Campisi, J., \& van Deursen, J. M. (2016). Senescent intimal foam cells are deleterious at all stages of atherosclerosis. Science, 354, 472-477.

Childs, B. G., Gluscevic, M., Baker, D. J., Laberge, R. M., Marquess, D., Dananberg, J., \& van Deursen, J. M. (2017). Senescent cells: An emerging target for diseases of ageing. Nature Reviews Drug Discovery, 16, 718-735.

Collado, M., Gil, J., Efeyan, A., Guerra, C., Schuhmacher, A. J., Barradas, M., ... Serrano, M. (2005). Tumour biology: Senescence in premalignant tumours. Nature, 436, 642.

Coppe, J. P., Desprez, P. Y., Krtolica, A., \& Campisi, J. (2010). The senescence-associated secretory phenotype: The dark side of tumor suppression. Annual Review of Pathology: Mechanisms of Disease, 5, 99-118.

Demaria, M., Ohtani, N., Youssef, S. A., Rodier, F., Toussaint, W., Mitchell, J. R., ... Campisi, J. (2014). An essential role for senescent cells in optimal wound healing through secretion of PDGF-AA. Developmental Cell, 31, 722-733.

Demaria, M., O'Leary, M. N., Chang, J., Shao, L., Liu, S., Alimirah, F., ... Campisi, J. (2017). Cellular senescence promotes adverse effects of chemotherapy and cancer relapse. Cancer Discovery, 7, 165-176.

Dimri, G. P., Lee, X., Basile, G., Acosta, M., Scott, G., Roskelley, C., ... Pereira-Smith, O. et al. (1995). A biomarker that identifies senescent human cells in culture and in aging skin in vivo. Proceedings of the National Academy of Sciences of the United States of America, 92, 9363-9367.

Fuhrmann-Stroissnigg, H., Ling, Y. Y., Zhao, J., McGowan, S. J., Zhu, Y., Brooks, R. W., ... Robbins, P. D. (2017). Identification of HSP90 inhibitors as a novel class of senolytics. Nature Communications, 8, 422.

Georgilis, A., Klotz, S., Hanley, C. J., Herranz, N., Weirich, B., Morancho, B., ... Gil, J. (2018). PTBP1-mediated alternative splicing regulates the inflammatory secretome and the pro-tumorigenic effects of senescent cells. Cancer Cell, 34(85-102), e109.

Gonzalez-Meljem, J. M., Apps, J. R., Fraser, H. C., \& Martinez-Barbera, J. P. (2018). Paracrine roles of cellular senescence in promoting tumourigenesis. British Journal of Cancer, 118, 1283-1288.

Gonzalez-Meljem, J. M., Haston, S., Carreno, G., Apps, J. R., Pozzi, S., Stache, C., ... Martinez-Barbera, J. P. (2017). Stem cell senescence drives age-attenuated induction of pituitary tumours in mouse models of paediatric craniopharyngioma. Nature Communications, 8, 1819.

Guerrero, A., Herranz, N., Sun, B., Wagner, V., Gallage, S., Guiho, R., ... Gil, J. (2019). Cardiac glycosides are broad-spectrum senolytics. Nature Metabolism, 1(11), 1074-1088. https://doi.org/10.1038/s4225 5-019-0122-z. 
Herranz, N., Gallage, S., Mellone, M., Wuestefeld, T., Klotz, S., Hanley, C. J., ... Gil, J. (2015). mTOR regulates MAPKAPK2 translation to control the senescence-associated secretory phenotype. Nature Cell Biology, 17, 1205-1217.

Herranz, N., \& Gil, J. (2018). Mechanisms and functions of cellular senescence. Journal of Clinical Investigation, 128, 1238-1246.

Hildebrand, D. G., Lehle, S., Borst, A., Haferkamp, S., Essmann, F., \& Schulze-Osthoff, K. (2013). alpha-Fucosidase as a novel convenient biomarker for cellular senescence. Cell Cycle, 12, 1922-1927.

Kuilman, T., \& Peeper, D. S. (2009). Senescence-messaging secretome: SMS-ing cellular stress. Nature Reviews Cancer, 9, 81-94.

Kurz, D. J., Decary, S., Hong, Y., \& Erusalimsky, J. D. (2000). Senescenceassociated (beta)-galactosidase reflects an increase in lysosomal mass during replicative ageing of human endothelial cells. Journal of Cell Science, 113(Pt 20), 3613-3622.

Lee, B. Y., Han, J. A., Im, J. S., Morrone, A., Johung, K., Goodwin, E. C., ... Hwang, E. S. (2006). Senescence-associated beta-galactosidase is lysosomal beta-galactosidase. Aging Cell, 5, 187-195.

Leenders, R. G., Damen, E. W., Bijsterveld, E. J., Scheeren, H. W., Houba, P. H., van der Meulen-Muileman, I. H., ... Haisma, H. J. (1999). Novel anthracycline-spacer-beta-glucuronide,-beta-glucoside, and -beta-galactoside prodrugs for application in selective chemotherapy. Bioorganic \& Medicinal Chemistry, 7, 1597-1610.

Melisi, D., Curcio, A., Luongo, E., Morelli, E., \& Rimoli, M. G. (2011). $D$-galactose as a vector for prodrug design. Current Topics in Medicinal Chemistry, 11, 2288-2298.

Munoz-Espin, D., Rovira, M., Galiana, I., Gimenez, C., Lozano-Torres, B., Paez-Ribes, M., ... Serrano, M. (2018). A versatile drug delivery system targeting senescent cells. EMBO Molecular Medicine, 10, e9355.

Munoz-Espin, D., \& Serrano, M. (2014). Cellular senescence: From physiology to pathology. Nature Reviews Molecular Cell Biology, 15, 482-496.

Ovadya, Y., \& Krizhanovsky, V. (2018). Strategies targeting cellular senescence. Journal of Clinical Investigation, 128, 1247-1254.

Salama, R., Sadaie, M., Hoare, M., \& Narita, M. (2014). Cellular senescence and its effector programs. Genes \& Development, 28, 99-114.

Schmitt, C. A., Fridman, J. S., Yang, M., Lee, S., Baranov, E., Hoffman, R. M., \& Lowe, S. W. (2002). A senescence program controlled by $p 53$ and p16INK4a contributes to the outcome of cancer therapy. Cell, 109, 335-346.

Tietze, L. F., Major, F., \& Schuberth, I. (2006). Antitumor agents: Development of highly potent glycosidic duocarmycin analogues for selective cancer therapy. Angewandte Chemie International Edition, 45, 6574-6577.

Tietze, L. F., \& Schmuck, K. (2011). Prodrugs for targeted tumor therapies: Recent developments in ADEPT, GDEPT and PMT. Current Pharmaceutical Design, 17, 3527-3547.

Tietze, L. F., Schuster, H. J., Krewer, B., \& Schuberth, I. (2009). Synthesis and biological studies of different duocarmycin based glycosidic prodrugs for their use in the antibody-directed enzyme prodrug therapy. Journal of Medicinal Chemistry, 52, 537-543.

Tietze, L. F., von Hof, J. M., Muller, M., Krewer, B., \& Schuberth, I. (2010). Glycosidic prodrugs of highly potent bifunctional duocarmycin derivatives for selective treatment of cancer. Angewandte Chemie International Edition, 49, 7336-7339.

Tordella, L., Khan, S., Hohmeyer, A., Banito, A., Klotz, S., Raguz, S., ... Gil, J. (2016). SWI/SNF regulates a transcriptional program that induces senescence to prevent liver cancer. Genes \& Development, 30, 2187-2198.

Triana-Martinez, F., Picallos-Rabina, P., Da Silva-Alvarez, S., Pietrocola, F., Llanos, S., Rodilla, V., ... Collado, M. (2019). Identification and characterization of Cardiac Glycosides as senolytic compounds. Nature Communications, 10, 4731.

Wang, L., Leite de Oliveira, R., Wang, C., Fernandes Neto, J. M., Mainardi, S., Evers, B., ... Bernards, R. (2017). High-throughput functional genetic and compound screens identify targets for senescence induction in cancer. Cell Reports, 21, 773-783.

Wang, Y., Chang, J., Liu, X., Zhang, X., Zhang, S., Zhang, X., ... Zheng, G. (2016). Discovery of piperlongumine as a potential novel lead for the development of senolytic agents. Aging (Albany NY), 8, 2915-2926.

Wirth, T., Schmuck, K., Tietze, L. F., \& Sieber, S. A. (2012). Duocarmycin Analogues Target Aldehyde Dehydrogenase 1 in Lung Cancer Cells. Angewandte Chemie. International Edition, 51, 2874-2877.

Yosef, R., Pilpel, N., Tokarsky-Amiel, R., Biran, A., Ovadya, Y., Cohen, S., ... Krizhanovsky, V. (2016). Directed elimination of senescent cells by inhibition of BCL-W and BCL-XL. Nature Communications, 7, 11190.

Zhu, Y., Tchkonia, T., Fuhrmann-Stroissnigg, H., Dai, H. M., Ling, Y. Y., Stout, M. B., ... Kirkland, J. L. (2016). Identification of a novel senolytic agent, navitoclax, targeting the Bcl-2 family of anti-apoptotic factors. Aging Cell, 15, 428-435.

Zhu, Y., Tchkonia, T., Pirtskhalava, T., Gower, A. C., Ding, H., Giorgadze, N., ... Kirkland, J. L. (2015). The Achilles' heel of senescent cells: From transcriptome to senolytic drugs. Aging Cell, 14, 644-658.

\section{SUPPORTING INFORMATION}

Additional supporting information may be found online in the Supporting Information section.

How to cite this article: Guerrero A, Guiho R, Herranz N, et al. Galactose-modified duocarmycin prodrugs as senolytics. Aging Cell. 2020;00:e13133. https://doi. org/10.1111/acel.13133 